\title{
The relationship of oral health literacy with oral health-related quality of life in a multi-racial sample of low-income female caregivers
}

Kimon Divaris ${ }^{1,2^{*}}$, Jessica Y Lee ${ }^{1,3}$, A Diane Baker ${ }^{1}$ and William F Vann $\mathrm{Jr}^{1}$

\begin{abstract}
Background: To investigate the association between oral health literacy $(\mathrm{OHL})$ and oral health-related quality of life (OHRQoL) and explore the racial differences therein among a low-income community-based group of female WIC participants.

Methods: Participants $(\mathrm{N}=1,405)$ enrolled in the Carolina Oral Health Literacy $(\mathrm{COHL})$ study completed the short form of the Oral Health Impact Profile Index (OHIP-14, a measure of OHRQoL) and REALD-30 (a word recognition literacy test). Socio-demographic and self-reported dental attendance data were collected via structured interviews. Severity (cumulative OHIP-14 score) and extent of impact (number of items reported fairly/very often) scores were calculated as measures of OHRQOL. OHL was assessed by the cumulative REALD-30 score. The association of OHL with OHRQoL was examined using descriptive and visual methods, and was quantified using Spearman's rho and zero-inflated negative binomial modeling.

Results: The study group included a substantial number of African Americans (AA $=41 \%$ ) and American Indians $(\mathrm{Al}=20 \%)$. The sample majority had a high school education or less and a mean age of 26.6 years. One-third of the participants reported at least one oral health impact. The OHIP-14 mean severity and extent scores were 10.6 [95\% confidence limits $(C L)=10.0,11.2]$ and $1.35(95 \% C L=1.21,1.50)$, respectively. OHL scores were distributed normally with mean (standard deviation, SD) REALD-30 of 15.8 (5.3). OHL was weakly associated with OHRQoL: prevalence $r h o=-0.14(95 \% \mathrm{CL}=-0.20,-0.08)$; extent $r h o=-0.14$ (95\% $C L=-0.19,-0.09)$; severity $r h o=-0.10$ (95\% $\mathrm{CL}=-0.16$, -0.05). "Low" OHL (defined as $<13$ REALD-30 score) was associated with worse OHRQoL, with increases in the prevalence of OHIP-14 impacts ranging from $11 \%$ for severity to $34 \%$ for extent. The inverse association of OHL with OHIP-14 impacts persisted in multivariate analysis: Problem Rate Ratio (PRR) $0.91(95 \% \mathrm{CL}=0.86,0.98)$ for one SD change in OHL. Stratification by race revealed effect-measure modification: Whites-PRR $=1.01$ (95\% CL $=0.91,1.11) ; \mathrm{AA}-\mathrm{PRR}=0.86(95 \% \mathrm{CL}=0.77,0.96)$.
\end{abstract}

Conclusions: Although the inverse association between OHL and OHRQOL across the entire sample was weak, subjects in the "low" OHL group reported significantly more OHRQoL impacts versus those with higher literacy. Our findings indicate that the association between OHL and OHRQoL may be modified by race.

Keywords: oral health literacy, oral health-related quality of life, OHIP-14, racial differences, effect measure modification

\footnotetext{
* Correspondence: divarisk@dentistry.unc.edu

'Department of Pediatric Dentistry. 228 Brauer Hall, CB\#7450, UNC School of Dentistry. University of North Carolina at Chapel Hill. Chapel Hill. North

Carolina, 27599, USA

Full list of author information is available at the end of the article
} 


\section{Background}

The importance of subjective measures of oral health is well-recognized in dental research [1-3]. Theoretical models have provided the framework that links clinical conditions with patient perceptions and impacts on their oral health-related quality of life (OHRQoL) [4,5]. Evidence shows that individuals' perceptions of their dental condition is closely related to OHRQoL, [6] and may confer greater impacts than the actual clinical conditions [1]. The United States (US) Surgeon General's report on Oral Health in America underscores and emphasizes the importance of OHRQoL, and its improvement on a population-level is defined as a goal [7]. For these reasons, subjective oral health $(\mathrm{SOH})$ instruments have been used to capture the multi-dimensional concept of OHRQoL $[8,9]$ and are used to quantify patient outcome experiences, monitor oral health status on national level, and identify dental public health goals $[10,11]$.

During this past decade the critical role of health literacy in medicine and public health has gained considerable attention $[12,13]$. The multi-level consequences of low health literacy have been reviewed extensively and include negative health behaviors, reduced utilization of preventive health services, and poorer adherence to therapeutic protocols $[14,15]$. Data from the most recent National Adult Literacy Survey (2003) indicate that an alarming proportion of US adults are functionally illiterate [16], and there exists evidence connecting low literacy with poorer health-related quality of life [17]. Health literacy is now considered an underlying cause of health disparities and has become a national health priority $[18,19]$.

Although much is known about health literacy in the medical context, little is known about oral health literacy (OHL) and its relationship to clinical conditions, patients' subjective assessments, and OHL's perceived impacts on daily life in the community. A working group of the National Institutes of Dental and Craniofacial Research (NIDCR) defined OHL as "the degree to which individuals have the capacity to obtain, process, and understand basic oral health information and services needed to make appropriate health decisions" [20]. Horowitz and Kleinman recently proposed that "oral health literacy is the new imperative for better oral health" as health literacy is now considered a determinant of health [21].

An accumulating body of evidence links low OHL with worse oral health outcomes such as oral health status [22,23], dental neglect [24] as well as sporadic dental attendance [25]. In a investigation among a group of Indigenous Australians, Parker and Jamieson [26] found that although low OHL was not associated with selfreported oral health status, it was associated with increased prevalence of OHIP-14 impacts (proportion of items reported fairly/very often). Noteworthy, in a recent study among child-caregiver dyads in the US, caregivers' OHL modified the association between children's oral health status and child OHRQoL impacts, with low-literacy caregivers reporting less impacts [27].

Previous pilot studies have explored the patterns of association between OHL and measures of OHRQoL using the Test of Functional Health Literacy in Dentistry (TOFHLiD) [28] and the Rapid Estimate of Adult Literacy in Dentistry (REALD-99) [29]. Interestingly, as in the Parker and Jamieson study, Richman and colleagues reported that while OHL was not associated with dental health status, higher OHL scores were significantly associated with less perceived OHIP-14 impacts, indicating better OHRQoL [29].

In the validation study of the short form of the REALD (REALD-30) among patients in a medical clinic setting, Lee et al [24] reported an inverse association of REALD-30 with OHIP-14 scores; however, the authors noted that because the data were collected on a convenience sample of health care-seeking subjects, future work is warranted on a larger, more diverse sample, as recommended by the NIDCR proposed research agenda [20]. To this end, the aims of the present study were to investigate the association between OHL and OHRQoL using REALD-30 in a large and more diverse and noncare seeking sample of subjects, and to explore any differences in this association between racial groups.

\section{Methods}

\section{Study population and recruitment}

This investigation relied upon interview data from the Carolina Oral Health Literacy (COHL) Project [30], a study exploring OHL in a low-income population of caregivers in the Women, Infants, and Children's Supplemental Nutrition Program (WIC) in North Carolina (NC). Non-random WIC sites in 7 counties in NC were selected using certain criteria including geographic region, rural/urban makeup, population demographics, active WIC clinics and established working relationships.

Study staff members were deployed in the selected WIC clinics and approached consecutive individuals to ask if they would answer eight questions from the study eligibility screening instrument. Eligibility criteria included being: a) the primary caregiver of a healthy (ASA I or II) and Medicaid-eligible infant/child 60 months old or younger, or expecting a newborn within the next 8 months, b) 18 years or older and c) Englishspeaking. Caregivers that met these criteria and agreed to participate were accompanied to a private area for a 30-minute in-person interview with one of the two trained study interviewers. Purposeful quota sampling [31] was employed to ensure that minority groups 
would be well-represented in the study sample. In this approach, individuals in pre-determined minority groups (African Americans and American Indians in the COHL study) are targeted preferentially and recruited into the study until adequate representation in the final sample is achieved. From 1,658 subjects that were screened and determined eligible 1,405 (85\%) participated and provided data in the domains of socio-demographic information, dental health and behaviors, OHRQoL, selfefficacy, and OHL. For the current analysis we excluded men $(n=49$ or $3.5 \%$ of total), Asians $(n=12$, or $0.9 \%)$, those who did not have English as their primary language at home ( $\mathrm{n}=79$ or $5.6 \%$ ), and those who had not yet reached age $18(\mathrm{n}=2$ or $0.1 \%)$. Therefore, our analytic sample included White, African American (AA) or American Indian (AI) female caregivers, whose primary language was English $(\mathrm{N}=1,278)$.

\section{Variable Measurements}

Additional demographic characteristics included age and education. Age was measured in years and coded as a quintile-categorical indicator variable. Education was coded as a four-level categorical variable where 1: did not finish high school, 2: high school or General Education Diploma (GED), 3: some technical education or some college, 4: college or higher education. Dental attendance was self-reported as the time since the last dental visit and coded as a four-level categorical variable where 1: < 1 year, 2: 12-23 months, 3: 2-5 years, 4: > 5 years or never.

OHRQoL impacts were assessed with the use of the short form of the Oral Health Impact Profile (OHIP-14) index [32]. Consistent with previous investigations [11], three OHIP-14 estimates were derived from subjects' responses: Severity (cumulative OHIP-14 score), prevalence (proportion of subjects reporting fairly/very often one or more items) and extent (number of items reported fairly/very often) of impacts were calculated as measures of OHRQoL. In terms of interpretation, the authors acknowledge Locker's critique that the OHIP may not fully satisfy the criteria for 'quality of life' measures [33], to be consistent with previous publications, however, have adopted the widely used term of OHRQoL in this manuscript.

OHL was measured with the previously validated word recognition test (REALD-30) [23]. The REALD-30 includes 30 words of dental context (e.g. fluoride, plaque, caries, halitosis, temporomandibular, etc.) arranged in order of increasing difficulty. The criteria used to determine word difficulty were based on word length, number of syllables, and difficult sound combinations, as well as results from 10 pre-test interviews that had been conducted prior to the REALD-30 validation study [23]. The study participant is asked to read each word out loud with one point given for each word that is pronounced correctly, resulting in a 0 -30 cumulative score where 0: lowest and 30: highest literacy. Although the REALD-30 is a word recognition test and may be capturing only some aspects of literacy skills, it has been shown to be highly correlated with functional health literacy [28] and to possess good psychometric properties [23]. Norms or thresholds for what constitutes "low OHL" have not been established, however in previous investigations $[27,34]$ a threshold of $<13$ on the 30 point REALD-30 scale was used to define a "low OHL" group.

\section{Analytical Strategy}

We used bivariate tabular methods to display the distribution of the three OHRQoL estimates (severity, prevalence and extent) by strata of socio-demographic variables. We calculated Spearman's correlation coefficients (rho) and 95\% confidence limits (CL; obtained with bootstrapping, $\mathrm{N}=1,000$ repetitions) to quantify the associations between OHL scores and prevalence, severity, and extent.

Although the inverse association between OHL and OHRQoL has been shown in previous investigations $[23,26]$, no information has been reported regarding the shape and gradient characteristics of this relationship. For this reason, we used polynomial smoothing functions (LPSF) and corresponding 95\% CL to illustrate the relationship between the OHL scores and OHIP-14 estimates. LPSF are non-parametric and data-adaptive functions [35,36] that are flexible in displaying an association without prior assumptions about its shape, gradient, or monotonicity, while minimizing biases from misspecification that could be introduced by traditional modeling applications. Further, to examine the association between "low" OHL and OHRQoL we used the < 13 REALD-30 score threshold, representing the lowest quartile of the distribution, to define the "low OHL" stratum. We obtained crude and adjusted differences and ratios of OHIP-14 impacts using Poisson models.

Because severity is the OHIP-14 estimate that arguably carries the most information (no items or scoring schemes are arbitrarily collapsed) and the entire range of the instrument scale (0-56) [11], we chose this measure for subsequent analytical iterations. To further quantify the association between OHL and severity, we used Zero-Inflated Negative Binomial modeling (ZINB). This analytical approach was used because of the distribution characteristics of severity, which followed a negative binomial type distribution with "excess zeros" (Figure 1).

The ZINB explicitly specifies two models that are fit simultaneously, one that models the "probability of zero" and one that models the count outcome, using a 


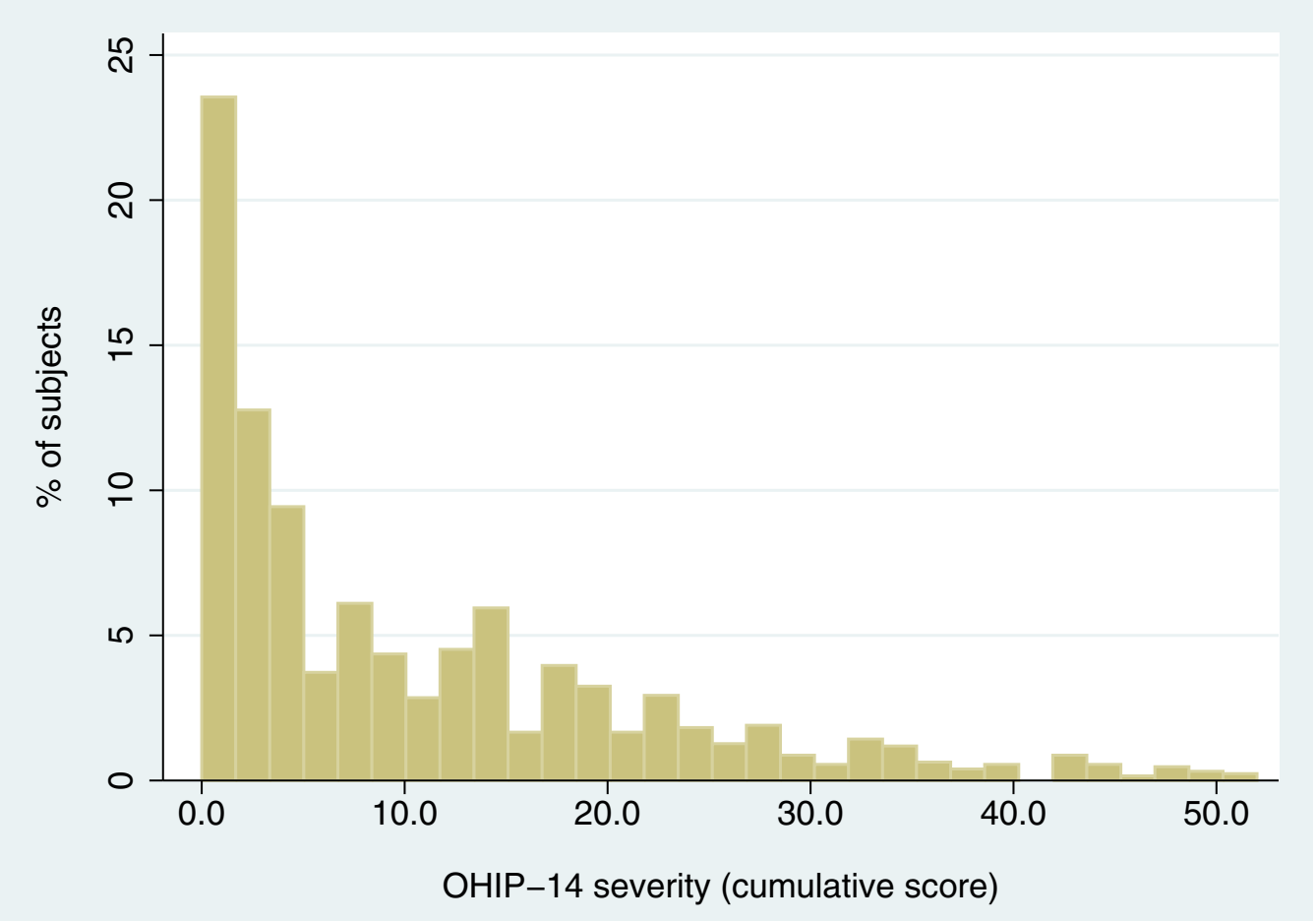

Figure 1 Distribution of OHIP-14 severity (cumulative score) among the female caregivers participating in the COHL study ( $\mathrm{N}=1,278$ ).

negative binomial distribution. These models have gained popularity in analyses of count outcomes with high proportion of zeros, but their selection and applicability can be data-specific $[37,38]$. For this reason and to determine the best fit, we considered other analytical approaches including the negative binomial (NB) and the zero inflated Poisson (ZIP) model. The appropriateness of ZINB versus the NB or the ZIP model was tested and confirmed with diagnostic model-fit statistics, using a Vuong test (ZINB favored over NB, $\mathrm{P}<0.05$ ) and a likelihood ratio test (ZINB favored over ZIP, $\mathrm{P}<0.05)$ [39].

The exponentiated coefficient of the negative binomial component of the model corresponds to a Prevalence Rate Ratio, which in this analysis we interpret as ratio of reported impacts (problems), or "Problem Rate Ratio" (PRR) as in a previous study [40]. To facilitate interpretation, we report model coefficients that correspond to one standard deviation change in OHL, which in our study was 5.3 units on the 30 unit REALD-30 scale. In other words, the PRR correspond to the change in reported cumulative OHIP-14 impacts that is associated with one standard deviation change in REALD-30 (expressed as ratio). Inclusion of confounders in the Poisson and the ZINB models was determined by likelihood ratio tests, comparing nested (reduced) models with the referent (full) model using a criterion of $\mathrm{P}<$ 0.1 . Interpretation of the model coefficients was based on effect estimation rather than hypothesis testing [41].

We employed three (race-specific) multivariate models to explore the possible heterogeneity of the association between OHL and OHRQoL between racial groups. Consistent with our aims, we considered race as an $a$ priori modifier of the examined association and therefore, these three models were identical to the "main effects" model but were restricted to strata of Whites, AAs and AIs. To determine whether race modified the association between literacy and quality of life, we compared these model-obtained race-specific estimates of the association between OHL and severity. The rationale for conducting comparisons of stratum-specific estimates as opposed to testing the hypothesis in the context of statistical interaction is based on the fact that the former approach does not assume covariate effecthomogeneity across racial groups. This could be a source of non-negligible bias when quantifying a weak main effect (e.g. OHL) in the presence of strong confounders (e.g. education), unless all potential interaction terms are included. To that end, we first conducted a global Wald $\mathrm{X}^{2}$ test of homogeneity or "a common PRR across racial groups" using a conservative criterion of $\mathrm{P}$ $<0.2$. We further examined post hoc differences in 
estimates between racial groups by calculating three pairwise homogeneity $Z$-scores $\left(Z_{\text {homog }}\right)$ using the formula: $Z_{\text {homog }}=\left|b_{x}-b_{y}\right| /\left(s_{x}{ }^{2}+s_{y}{ }^{2}\right)^{1 / 2}$, where $b_{x / y / z}$ and $\mathrm{se}_{\mathrm{x} / \mathrm{y} / \mathrm{z}}$ are the ZINB model-obtained race-specific coefficients and standard errors respectively [42]. Two-tailed $\mathrm{P}$-values corresponding to the Z-scores were obtained using the normal distribution function of the Stata 12.0 (StataCorp LP, College Station, TX) statistical program. A $\mathrm{P}<0.05$ criterion was used for the pairwise tests.

\section{Results}

The demographic characteristics of our final analytic sample $(\mathrm{N}=1,280)$ with corresponding OHIP-14 prevalence, extent, and severity scores are presented in Table 1. Participants' mean age in years was 26.6 (median = 25). Sixty percent had a high school education or less. Seventy-five percent reported a dental visit within the last two years.

The OHL score was distributed normally [30] with a mean (SD) REALD-30 of 15.8 (5.3), with $25 \%$ of participants $(\mathrm{N}=316)$ scoring less than 13 , classified as "low OHL". Pronounced OHL gradients were noted relative to education as follows: less than high school-13.0 (4.8), high school or GED-15.0 (4.9), some technical or college-18.0 (4.7) and college degree or higher-20.1 (4.8).
Differences by race were also evident: whites-17.4 (4.9), AA-15.3 (5.1), AI-13.7 (5.3). The mean OHIP-14 severity and extent scores were $10.6(95 \% \mathrm{CI}=10.0,11.2)$ and 1.35 (95\% CI $=1.21,1.50)$, respectively. Thirtyseven percent reported at least one oral health impact fairly or very often (prevalence), while AIs had the highest severity score. A strong gradient was found with decreasing age and OHIP-14 scores. Some age and racial differences were noted, with older subjects and AIs reporting more impacts.

OHL showed weak correlations with all three OHIP14 estimates: prevalence rho $=-0.14(95 \% \mathrm{CI}=-0.20$, $-0.08)$, extent rho $=-0.14(95 \% \mathrm{CI}=-0.19,-0.09)$, and severity rho $=-0.10(95 \% \mathrm{CI}=-0.16,-0.05)$. These bivariate associations are illustrated in Figures 2a, b, and 2c with local polynomial smoothing functions and $95 \%$ confidence intervals. In these illustrations the inverse, non-linear association between OHL and the OHRQoL estimates was evident. Although the negative gradient was more apparent for prevalence, the inverse relationship of all three OHRQoL measures with OHL was more "profound" at the lower end of the OHL range. This was confirmed by the contrast of the "low" versus the "high OHL" group (Table 2), where the former group had consistently worse OHRQoL estimates. "Low

Table 1 Distribution of oral health-related quality of life (OHRQoL) measures [OHIP-14 estimates and corresponding 95\% confidence limits (CL)] by demographic characteristics among the Carolina Oral Health Literacy study participants $(\mathrm{N}=\mathbf{1 , 2 7 8 )}$

\begin{tabular}{|c|c|c|c|c|c|}
\hline & \multirow[b]{2}{*}{$\mathrm{N}$} & \multirow[b]{2}{*}{ (\%) } & \multicolumn{3}{|c|}{ Subjective oral health impacts estimates (OHIP14) } \\
\hline & & & $\begin{array}{l}\text { Prevalence } \\
\text { (95\% CL) }\end{array}$ & $\begin{array}{l}\text { Severity } \\
(95 \% \mathrm{CL})\end{array}$ & $\begin{array}{c}\text { Extent } \\
(95 \% \mathrm{CL})\end{array}$ \\
\hline \multicolumn{6}{|l|}{ Race } \\
\hline White & 503 & 39 & $36.6(32.4,40.8)$ & $10.6(9.6,11.6)$ & $1.39(1.15,1.62)$ \\
\hline African American & 522 & 41 & $34.7(30.6,38.8)$ & $10.4(9.4,11.3)$ & $1.24(1.04,1.45)$ \\
\hline American Indian & 253 & 20 & $39.1(33.1,45.2)$ & $11.2(9.8,12.6)$ & $1.53(1.19,1.87)$ \\
\hline \multicolumn{6}{|l|}{ Education } \\
\hline Less than high school & 305 & 24 & $49.5(43.9,55.2)$ & $13.6(12.1,15.0)$ & $2.10(1.74,2.45)$ \\
\hline High school diploma/GED & 479 & 37 & $35.1(30.8,39.4)$ & $10.3(9.3,11.3)$ & $1.23(1.01,1.45)$ \\
\hline Some technical or college & 429 & 34 & $31.5(27.1,35.9)$ & $9.4(8.5,10.4)$ & $1.10(0.88,1.31)$ \\
\hline College or higher & 65 & 5 & $15.4(6.4,24.4)$ & $7.1(4.9,9.2)$ & $0.45(0.15,0.74)$ \\
\hline \multicolumn{6}{|l|}{ Dental attendance } \\
\hline$<12$ months & 726 & 57 & $34.7(31.2,38.2)$ & $10.4(9.6,11.2)$ & $1.30(1.12,1.48)$ \\
\hline 12-23 months & 217 & 17 & $31.3(25.1,37.6)$ & $9.5(8.0,11.0)$ & $1.24(0.91,1.57)$ \\
\hline $2-5$ years & 177 & 14 & $45.8(38.4,53.2)$ & $11.2(9.5,12.9)$ & $1.52(1.16,1.88)$ \\
\hline$>5$ years & 151 & 12 & $39.9(32.2,47.6)$ & $12.6(10.7,14.4)$ & $1.58(1.11,2.04)$ \\
\hline Age (years; quintiles) & & Mean(SD) & & & \\
\hline Entire sample & 1,278 & 26.6(6.9) & & & \\
\hline Q1 range: 18.0, 20.9 & 256 & 19.6(0.8) & $28.9(23.3-34.5)$ & $8.3(7.1-9.6)$ & $1.04(0.77,1.32)$ \\
\hline Q2 range: $20.9,23.4$ & 256 & $22.1(0.7)$ & $40.6(34.6-46.7)$ & $11.2(9.8-12.5)$ & $1.47(1.16,1.79)$ \\
\hline Q3 range: $23.4,26.5$ & 255 & $24.8(0.9)$ & $34.5(28.6-40.4)$ & $10.4(9.1-11.7)$ & $1.22(0.92,1.53)$ \\
\hline Q4 range: $26.5,30.9$ & 256 & 28.6(1.3) & $37.1(31.2-43.1)$ & $10.8(9.5-12.1)$ & $1.35(1.04,1.66)$ \\
\hline Q5 range: $30.9,65.6$ & 255 & $37.7(6.1)$ & $40.4(34.3-46.5)$ & $12.5(10.8-14.1)$ & $1.69(1.32,2.06)$ \\
\hline
\end{tabular}



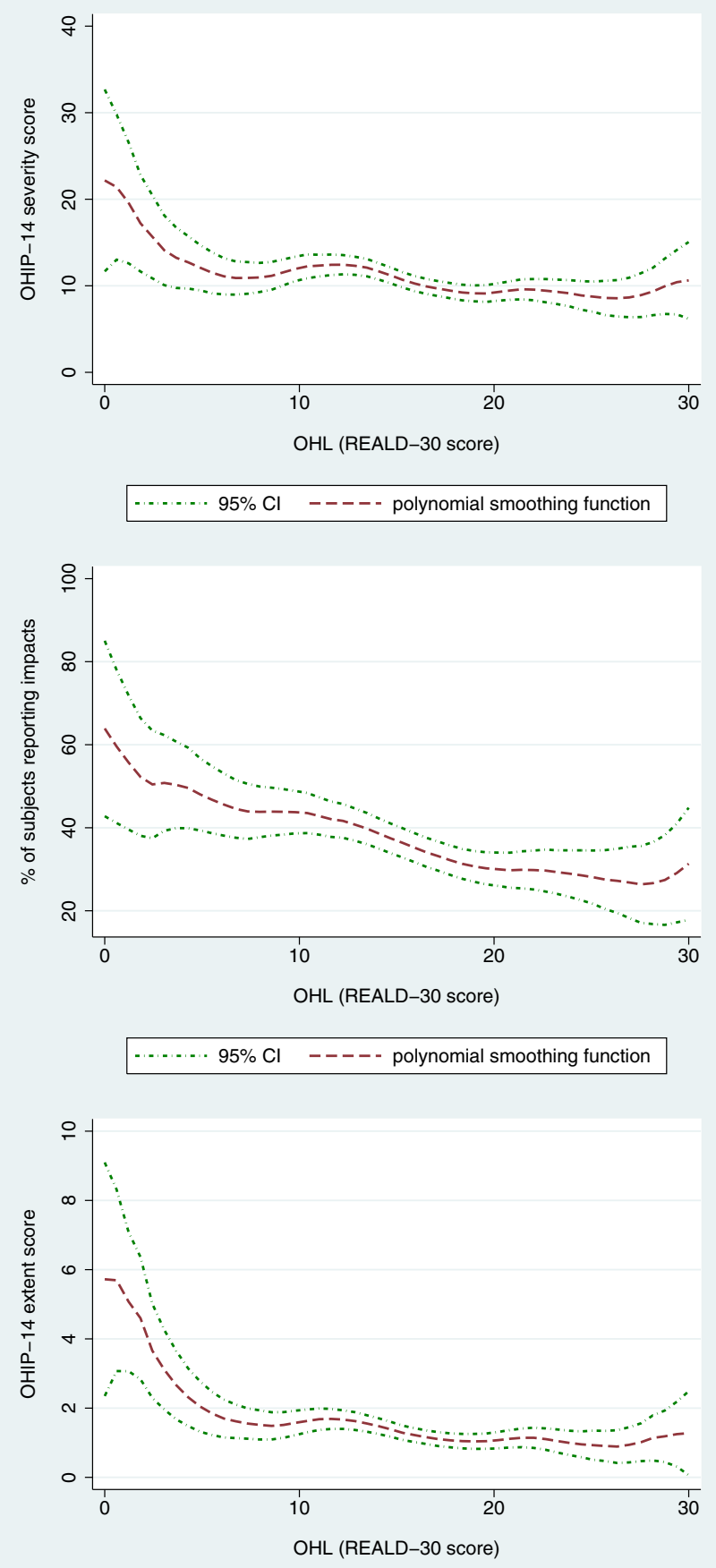

-...... 95\% Cl - - - polynomial smoothing function

Figure 2 Relationship between OHL and oral health related quality of life estimates [OHIP-14 severity (a), prevalence (b) and extent (c)] illustrated by polynomial smoothing functions and corresponding $95 \%$ confidence limits, among the female caregivers participating in the COHL study $(\mathrm{N}=1,278)$. 
Table 2 Oral health-related quality of life (OHRQoL) differences [mean difference and prevalence ratios (PR) with corresponding 95\% confidence limits (CL)] between participants with "low" (< 13 REALD-30; referent category) and "high" ( $\geq 13$ REALD-30) oral health literacy in the Carolina Oral Health Literacy study $(\mathbf{N}=1,278)$

\begin{tabular}{|c|c|c|c|c|c|}
\hline \multirow{3}{*}{$\begin{array}{l}\text { OHRQoL } \\
\text { (OHIP-14 es }\end{array}$} & \multirow{2}{*}{$\begin{array}{c}\text { “Low" literacy } \\
\text { (< } 13 \text { REALD-30) } \\
\text { N = } 316(25 \%)\end{array}$} & \multirow{2}{*}{$\begin{array}{l}\text { “High" literacy } \\
\text { ( } \geq 13 \text { REALD-30) } \\
\mathrm{N}=962(75 \%)\end{array}$} & \multicolumn{2}{|c|}{ Difference $^{1}$ [mean $\left.(95 \% \mathrm{CL})\right]$} & \multirow{2}{*}{$\begin{array}{c}\text { Prevalence Ratio }^{1} \text { [(PR (95\% CL)] } \\
\text { Adjusted }^{2}\end{array}$} \\
\hline & & & Crude & Adjusted $^{2}$ & \\
\hline & & & & & \\
\hline Prevalence & $45.3(39.7,50.8)$ & $33.4(30.4,36.4)$ & $11.9(0.04,0.20)$ & $7.4(-1.4,16.2)$ & $1.17(1.00,1.37)$ \\
\hline Severity & $12.4(11.0,13.8)$ & $10.1(9.4,10.7)$ & $2.3(1.9,2.8)$ & $1.2(0.7,1.6)$ & $1.11(1.07,1.16)$ \\
\hline Extent & $1.87(1.52,2.22)$ & $1.19(1.04,1.33)$ & $0.68(0.52,0.85)$ & $0.36(0.19,0.54)$ & $1.34(1.20,1.50)$ \\
\hline
\end{tabular}

1: Mean differences and ratios of OHIP-14 impacts were calculated using the "high literacy" category as referent.

2: Adjusted differences and ratios were obtained using a Poisson model controlling for race, age, education level and dental attendance.

OHL" was associated with significant absolute and relative increases in all OHRQoL dimensions, with relative prevalence estimates ranging from $+11 \%$ for severity to $+34 \%$ for extent.

Multivariate analysis adjusting for age, race, and education revealed that the weak inverse association between OHL and severity across the entire sample persisted: PRR $=0.91$ (95\% CL $=0.86,0.98)$. Table 2 presents estimates obtained from the stratified (racespecific) multivariate models, where: Whites-PRR = $1.01(95 \% \mathrm{CL}=0.91,1.11), \mathrm{AA}-\mathrm{PRR}=0.86(95 \% \mathrm{CL}=$ $0.77,0.96)$ and $A I-P R R=0.92(95 \% C L=0.80,1.05)$. By comparing these estimates ensemble we rejected the assumption of homogeneity (Wald $\mathrm{X}^{2}=4.6$; degrees of freedom $=2 ; \mathrm{P}<0.2)$. Subsequent pairwise comparisons of the race-specific estimates confirmed that the measures of association among AAs and Whites departed from homogeneity $\left(Z_{\text {homog }}=2.06 ; \mathrm{P}<0.05\right)$. In fact, no association between OHL and OHIP-14 severity was found among Whites whereas weak associations were found among AAs and AIs.

\section{Discussion}

This investigation provides the first report of the association between OHL and OHRQoL (as measured by OHIP-14) in a multi-racial community-based sample. This study was restricted to a non-probability sample of low-income female caregivers participating in the WIC program in NC; however, we believe that this homogeneity is advantageous because strong income-gradients have been identified in oral health impacts on the population level $[43,44]$. Moreover, recruitment of subjects from a non-dental clinical environment reduces the potential for selection bias and, within the limitations of the sampling procedures and target population, increases the generalizability of our findings. It is noteworthy but not surprising that the OHL levels in this study were considerably lower than those reported for dental patients seeking care in private practice [REALD30 (SD): 23.9 (1.3)] [22] or a dental school setting [20.7
(5.5)] [45], and comparable to those found among a community-based sample of indigenous Australians [15.0 (7.8)] [26].

It has been acknowledged that minority individuals and those towards the lowest end of the literacy distribution may be underrepresented in oral health research [46] and this can be even more exacerbated in literacy investigations. Interestingly, the most profound negative gradients between OHL and OHRQoL measures were observed at the lower end of the OHL spectrum, with subjects scoring $<13$ on the 30 -point REALD-30 scale reporting significantly more OHRQoL impacts versus those with higher literacy. This finding is consistent with conceptual frameworks that consider skills such as conceptual knowledge and OHL as pre-requisites of appropriate decision-making [47]. It is likely that OHL exerts strong influences on oral health-related outcomes when below a certain threshold, but it may be a less impactful determinant at higher levels.

The high representation of AAs and AIs that were enrolled in COHL offered us an opportunity to examine for any underlying heterogeneity in the association of $\mathrm{OHL}$ with $\mathrm{SOH}$ between racial groups. We found a weak negative association between OHL and OHIP-14 severity for AAs and AIs, but not Whites. While AAs have been shown to report worse OHIP scores in the US [10] and patterns of OHRQoL changes have been shown to differ by race $[48,49]$, this finding warrants further investigation; race may be a proxy of unmeasured mediating factors between $\mathrm{OHL}$, oral health status, and perceived impacts [50]. The fact that the dimensionality of OHRQoL [8] may differ between diverse populations or ethnic groups may amplify this phenomenon; therefore, we acknowledge the limitation of our analytical sample that was restricted to low-income WIC-participating female caregivers. Replication of our main as well as race-specific findings should be undertaken on a population-based representative sample.

Lawrence et al [51] recently demonstrated that OHIP14 scores show good correlation with clinical oral health 
status, independent of gender and socioeconomic inequalities in oral health. Among our community-based caregivers, the prevalence of oral health impacts (36.5\%) was higher compared to nationally representative samples from other studies including the US (15.3\%) [10], Australia (dentate subjects-18.2\%), United Kingdom (dentate subjects-15.9\%) [11] and New Zealand (23.4\%) [51]. However, the extent and severity estimates reported here are lower compared to these samples. One possible interpretation of this finding is that our study group was limited to young, low-income, poorly educated, WIC participants with relatively low education. The young mean age (26.6 years) may explain the low severity and extent estimates while the low-income and low-education level status may explain the high prevalence of at least one impact reported as fairly/very often.

Considering the high prevalence of impacts revealed in the study population, the significance of lower OHL is demonstrative. Using our "main effects" model coefficients, we estimate that a one standard deviation increase in OHL (5.3 REALD-30 units) corresponds to a 9\% decrease in OHIP-14 severity [PRR $(95 \% \mathrm{CL})=0.91$ $(0.86,0.98)]$, whereas (using race-specific estimates from Table 3) this decrease is more pronounced (14\%) among AA [PRR $(95 \% \mathrm{CL})=0.86(0.77,0.96)]$. On the other hand, this finding provides a foundation to consider interventions to enhance OHL, or rather improve the readability of written materials and accessibility to dental services to an appropriate literacy level [30]. It remains uncertain whether improvement in OHL is feasible and if so, whether this would lead to better oral health status and subjective oral health. Although education and income arguably remain the strongest correlates of oral health and disease, and literacy is one of numerous other distal determinants, OHL may be part of causal mechanisms that lead to worse oral health [21]. Accumulating evidence linking poor OHL with adverse oral health outcomes among caregivers [24] and their young children $[27,34]$ supports the introduction

\begin{tabular}{|c|c|c|}
\hline & $\mathrm{PRR}^{2}$ & $95 \% \mathrm{CL}$ \\
\hline Entire sample & 0.91 & $0.86,0.98$ \\
\hline \multicolumn{3}{|l|}{ Race } \\
\hline White & 1.01 & $0.91,1.11$ \\
\hline African American & 0.86 & $0.77,0.96$ \\
\hline American Indian & 0.92 & $0.80,1.05$ \\
\hline
\end{tabular}

1: Zero-inflated negative binomial model, including terms for age, education level and dental attendance.

2: Estimates corresponds to the relative change in OHIP-14 cumulative score for one standard deviation increase in $\mathrm{OHL}$. and implementation of rapid OHL screening tools [52] in clinical practice, dental research and public health surveillance. Moreover, we suggest that more studies exploring the association between OHL and OHRQoL be undertaken in multi-racial community based samples to confirm or reject this study's finding of effect measure modification by race.

\section{Conclusions}

We found a high prevalence of perceived oral health impacts in this sample of low-income female WIC participants. Although the inverse association between OHL and OHRQoL across the entire sample was weak, subjects in the "low" OHL group reported significantly more OHRQoL impacts versus those with higher literacy. Within the limitations of our study among low-income female caregivers, our findings indicate that the association between OHL and OHRQoL appears to be modified by race.

\section{Acknowledgements}

The COHL Project is supported by the NIDCR Grant RO1DE018045.

\section{Author details}

'Department of Pediatric Dentistry. 228 Brauer Hall, CB\#7450, UNC School of Dentistry. University of North Carolina at Chapel Hill. Chapel Hill. North Carolina, 27599, USA. ²Department of Epidemiology. 228 Brauer Hall, CB\#7450, UNC School of Dentistry. University of North Carolina at Chapel Hill. Chapel Hill. North Carolina, 27599, USA. ${ }^{3}$ Department of Health Policy and Management. CB\#7411. University of North Carolina at Chapel Hill. Chapel Hill. North Carolina, 27599, USA.

\section{Authors' contributions}

$\mathrm{KD}$ conducted the data analysis and prepared the first draft of the manuscript. $J$ conceived the study, overviewed the data analysis, contributed to the interpretation of results and assisted in preparation of the first draft of the manuscript. ADB participated in data collection, and critically revised the manuscript. WFV contributed to the interpretation of results and critically revised the manuscript. All authors read and approved the final manuscript.

\section{Competing interests}

The authors declare that they have no competing interests.

Received: 6 July 2011 Accepted: 1 December 2011

Published: 1 December 2011

\section{References}

1. Lee IC, Shieh TY, Yang YH, Tsai CC, Wang KH: Individuals' perception of oral health and its impact on the health-related quality of life. J Oral Rehabil 2007, 34:79-87.

2. Allen PF: Assessment of oral health related quality of life. Health Qual Life Outcomes 2003, 1:40.

3. Gift HC, Atchison KA: Oral health, health, and health-related quality of life. Med Care 1995, 33(11 Suppl):NS57-77.

4. Locker D: Measuring oral health: a conceptual framework. Community Dent Health 1988, 5:3-18.

5. Wilson IB, Cleary PD: Linking clinical variables with health-related quality of life. A conceptual model of patient outcomes. JAMA 1995, 273:59-65.

6. Brennan DS, Spencer AJ: Mapping oral health related quality of life to generic health state values. BMC Health Serv Res 2006, 6:96.

7. Department of Health and Human Services: Oral Health in America: A Report of the Surgeon General. Rockville, Md: National Institute of Dental and Craniofacial Research, National Institutes of Health, US Dept of Health 
and Human Services; 2000:7:158-168[http://www2.nidcr.nih.gov/sgr/ sgrohweb/home.htm].

8. Montero J, Bravo M, Vicente MP, Galindo MP, López JF, Albaladejo A: Dimensional structure of the oral health-related quality of life in healthy Spanish workers. Health Qual Life Outcomes 2010, 8:24.

9. John MT: Exploring dimensions of oral health-related quality of life using experts' opinions. Qual Life Res 2007, 16:697-704.

10. Sanders AE, Slade GD, Lim S, Reisine ST: Impact of oral disease on quality of life in the US and Australian populations. Community Dent Oral Epidemiol 2009, 37:171-81.

11. Slade GD, Nuttall N, Sanders AE, Steele JG, Allen PF, Lahti S: Impacts of oral disorders in the United Kingdom and Australia. Br Dent J 2005, 198:489-493.

12. Protheroe J, Wallace $L S$, Rowlands G, DeVoe JE: Health literacy: setting an international collaborative research agenda. BMC Fam Pract 2009, 10:51.

13. Nutbeam D: The evolving concept of health literacy. Soc Sci Med 2008, 67:2072-2078.

14. Dewalt DA, Berkman ND, Sheridan S, Lohr KN, Pignone MP: Literacy and health outcomes: a systematic review of the literature. J Gen Intern Med 2004, 19:1228-1239.

15. Andrus MR, Roth MT: Health literacy: a review. Pharmacotherapy 2002, 22:282-302.

16. Kutner M, Greenberg E, Jin Y, Boyle B, Hsu Y, Dunleavy E: Literacy in Everyday Life: Results from the 2003 National Assessment of Adult Literacy. Washington, DC: US Department of Education; 2007, 2007-4800 [http://nces.ed.gov/Pubs2007/2007480.pdf], NCES.

17. Wallace $L S$, Rogers ES, Weiss BD: Relationship between health literacy and health-related quality of life among Tennesseans. Tenn Med 2008, 101:35-39.

18. Baker DW: The meaning and the measure of health literacy. J Gen Intern Med 2006, 21:878-883.

19. Patrick DL, Lee RS, Nucci M, Grembowski D, Jolles CZ, Milgrom P: Reducing oral health disparities: a focus on social and cultural determinants. BMC Oral Health 2006, 6(Suppl 1):S4.

20. National Institute of Dental and Craniofacial Research, National Institute of Health, U.S. Public Health Service, Department of Health and Human Services: The invisible barrier: literacy and its relationship with oral health. A report of a workgroup sponsored by the National Institute of Dental and Craniofacial Research, National Institute of Health, U.S. Public Health Service, Department of Health and Human Services. $J$ Public Health Dent 2005, 65:174-182.

21. Horowitz AM, Kleinman DV: Oral health literacy: the new imperative to better oral health. Dent Clin North Am 2008, 52:333-344.

22. Jones M, Lee JY, Rozier RG: Oral health literacy among adult patients seeking dental care. J Am Dent Assoc 2007, 138:1199-1208.

23. Lee JY, Rozier RG, Lee SY, Bender D, Ruiz RE: Development of a word recognition instrument to test health literacy in dentistry: the REALD-30 - a brief communication. J Public Health Dent 2007, 67:94-98.

24. Lee JY, Divaris K, Baker D, Rozier RG, Vann WF Jr: The Relationship of Oral Health Literacy with Oral Health Status and Dental Neglect. Am J Public Health 2011, e1-e7.

25. Sabbahi DA, Lawrence HP, Limeback H, Rootman I: Development and evaluation of an oral health literacy instrument for adults. Community Dent Oral Epidemiol 2009, 37:451-462.

26. Parker EJ, Jamieson LM: Associations between Indigenous Australian oral health literacy and self-reported oral health outcomes. BMC Oral Health 2010, 10:3.

27. Divaris K, Lee JY, Baker AD, Vann WF Jr: Caregivers' oral health literacy and their young children's oral health related quality of life. Acta Odont Scand.

28. Gong DA, Lee JY, Rozier RG, Pahel BT, Richman JA, Vann WF Jr: Development and testing of the Test of Functional Health Literacy in Dentistry (TOFHLiD). J Public Health Dent 2007, 67:105-112.

29. Richman JA, Lee JY, Rozier RG, Gong DA, Pahel BT, Vann WF Jr: Evaluation of a word recognition instrument to test health literacy in dentistry: the REALD-99. J Public Health Dent 2007, 67:99-104.

30. Lee JY, Divaris K, Baker D, Rozier RG, Lee SY, Vann WF Jr: Oral health literacy levels among a low-income WIC population. J Public Health Dent 2011, 71:152-160.

31. Kalton G: Models in the practice of survey sampling. International Statistical Review 1983, 51:175-188.
32. Slade GD: Derivation and validation of a short-form oral health impact profile. Community Dent Oral Epidemiol 1997, 25:284-290.

33. Locker D, Allen F: What do measures of 'oral health-related quality of life' measure? Community Dent Oral Epidemiol 2007, 35:401-411.

34. Vann WF Jr, Lee JY, Baker D, Divaris K: Oral health literacy among female caregivers: impact on oral health outcomes in early childhood. $J$ Dent Res 2010, 89:1395-1400.

35. Fan J, Gijbels I: Local Polynomial Modelling and its Applications. London: Chapman \& Hall; 1996, 14-22.

36. Cleveland WS: Robust locally weighted regression and smoothing scatterplots. Journal of the American Statistical Association 1979, 74:829-836.

37. Lee AH, Wang K, Scott JA, Yau KK, McLachlan GJ: Multi-level zero-inflated poisson regression modelling of correlated count data with excess zeros. Stat Methods Med Res 2006, 15:47-61.

38. Lewsey JD, Thomson WM: The utility of the zero-inflated poisson and zero-inflated negative binomial models: a case study of cross-sectional and longitudinal DMF data examining the effect of socio-economic status. Community Dent Oral Epidemiol 2004, 32:183-189.

39. Vuong QH: Likelihood Ratio Tests for Model Selection and Non-Nested Hypotheses. Econometrica 1989, 57:307-333.

40. John MT, Slade GD, Szentpétery A, Setz JM: Oral health-related quality of life in patients treated with fixed, removable, and complete dentures 1 month and 6 to 12 months after treatment. Int J Prosthodont 2004, 17:503-511.

41. Poole C: Beyond the confidence interval. Am J Public Health 1987, 77:195-199.

42. Greenland S, Rothman KJ: Introduction to stratified analysis. In Modern epidemiology. Edited by: Rothman KJ, Greenland S, Lash TL. New York: Lippincott, Williams and Wilkins; 2008:258-282.

43. Sanders AE, Slade GD, John MT, Steele JG, Suominen-Taipale AL, Lahti S, Nuttall NM, Allen PF: A cross-national comparison of income gradients in oral health quality of life in four welfare states: application of the Korpi and Palme typology. J Epidemiol Community Health 2009, 63:569-574.

44. Sanders AE, Slade GD, Turrell G, John Spencer A, Marcenes W: The shape of the socioeconomic-oral health gradient: implications for theoretical explanations. Community Dent Oral Epidemiol 2006, 34:310-319.

45. Miller E, Lee JY, DeWalt DA, Vann WF Jr: Impact of caregiver literacy on children's oral health outcomes. Pediatrics 2010, 126:107-114.

46. Simon MA, Dong X, Nonzee N, Bennett CL: Heeding our words: complexities of research among low-literacy populations. J Clin Oncol 2009, 27:1938-1940.

47. Macek MD, Haynes D, Wells W, Bauer-Leffler S, Cotten PA, Parker RM: Measuring conceptual health knowledge in the context of oral health literacy: preliminary results. J Public Health Dent 2010, 70:197-204.

48. Hyde S, Satariano WA, Weintraub JA: Welfare dental intervention improves employment and quality of life. J Dent Res 2006, 85:79-84.

49. Gilbert GH: Racial and socioeconomic disparities in health from population-based research to practice-based research: the example of oral health. J Dent Educ 2005, 69:1003-1014.

50. Manly JJ: Deconstructing race and ethnicity: implications for measurement of health outcomes. Med Care 2006, 44(11 Suppl 3):S10-6.

51. Lawrence HP, Thomson WM, Broadbent JM, Poulton R: Oral health-related quality of life in a birth cohort of 32-year olds. Community Dent Oral Epidemiol 2008, 36:305-316.

52. Stucky BD, Lee JY, Lee SY, Rozier RG: Development of the Two-Stage Rapid Estimate of Adult Literacy in Dentistry. Community Dent Oral Epidemiol 2011.

doi:10.1186/1477-7525-9-108

Cite this article as: Divaris et al:: The relationship of oral health literacy with oral health-related quality of life in a multi-racial sample of lowincome female caregivers. Health and Quality of Life Outcomes 2011 9:108. 